\title{
Nonlinear relativistic optics in the single cycle, single wavelength regime and kilohertz repetition rate
}

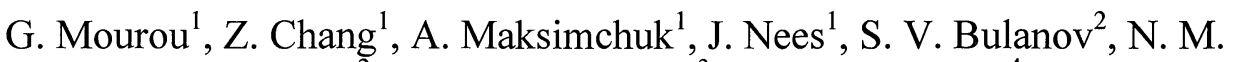 \\ Naumova ${ }^{2}$, V. Yu. Bychenkov ${ }^{3}$, T. Zh. Esirkepov ${ }^{4}$, \\ F. Pegoraro 5 and H. Ruhl ${ }^{6}$ \\ ${ }^{1}$ Center for Ultrafast Optical Science, University of Michigan, Ann Arbor, Michigan 48109-2099 \\ ${ }^{2}$ General Physics Institute of the Russian Academy of Sciences, Moscow, Russia 117942 \\ ${ }^{3}$ P. N. Lebedev Physics Institute of the Russian Academy of Sciences, Moscow, Russia 117924 \\ ${ }^{4}$ Moscow Institute for Physics and Technology, Moscow Region, Russia 141700 \\ ${ }^{5}$ Pisa University and Instituto Nazionale Fisica della Materia, Pisa, Italy \\ ${ }^{6}$ Max Born Institute, Berlin, Germany
}

\begin{abstract}
Pulses of few optical cycles, focused on one wavelength with relativistic intensities can be produced at a kilohertz repetition rate. By properly choosing the plasma and laser parameters, relativistic nonlinear effects, such as channeling and electron and ion acceleration to tens of megaelectronvolts are demonstrated.
\end{abstract}

\section{INTRODUCTION}

Laser intensity in the relativistic regime, i.e. greater than $10^{18} \mathrm{~W} / \mathrm{cm}^{2}$ for $1 \mu \mathrm{m}$ light $^{1}$, has opened new frontiers in physics. At this intensity level electrons acquire a quiver energy greater than $0.5 \mathrm{MeV}$, corresponding to the rest mass of the electron. The relativistic character of electrons is dominated by a mass increase and a large ponderomotive force $(\vec{v} \times \vec{B})$ where $\vec{v}$ is the quiver velocity of the electrons and $\vec{B}$ the light's magnetic field. In interaction of a high-intensity light with matter a host of novel effects have been demonstrated: the production of high energy electron and ion beams ${ }^{2,3}$, the generation of the directional $\gamma$-ray pulses ${ }^{4}$, the demonstration of relativistic harmonics from solids ${ }^{5}$, relativistic self-focusing ${ }^{6}$ and nonlinear Thomson scattering ${ }^{7}$, etc. The lasers involved in these studies although more compact than its predecessors are still very large and expensive with energy in the joule level, at repetition rate from $0.01 \mathrm{~Hz}$ to $10 \mathrm{~Hz}$ and with pulse duration greater than $100 \mathrm{fs}$. Thanks to the progresses in short pulse generation and the application of deformable mirrors for beam focusing, we have recently shown ${ }^{8}$ that it is possible to produce relativistic intensities at a kilohertz repetition rate. The laser

CP611, Superstrong Fields in Plasmas: Second Int'l. Conf., edited by M. Lontano et al. (c) 2002 American Institute of Physics 0-7354-0057-1/02/\$19.00 
pulse energy is in the millijoule range, with sub-ten femtosecond duration, i.e. in the single-cycle regime and a focused spot size of one wavelength dimension.

Because of their very short Rayleigh range of the order of $1 \mu \mathrm{m}$, these pulses would have only limited applications, such as harmonic generation from solid's interface. To extend their usage it is crucial to increase their interaction distance with the plasma. This condition is necessary, for instance, in particle acceleration (electron, positron, ion). In this paper we demonstrate numerically that in a situation, similar to single-mode graded index optics, if the laser numerical aperture, $N A$, is matched with the relativistic waveguide numerical aperture, set by the laser power and the plasma frequency, singlemode propagation of the relativistic pulse, over many Rayleigh ranges can be obtained.

This important result opens the door to a number of exciting prospects including: i) the possibility to produce few-femtosecond-duration, tens-of-MeV electron/positron/ion bursts, with a very compact system at a $\mathrm{kHz}$ repetition rate; ii) femtosecond injectors for electron/positron accelerators; iii) the production of para- and ortho-positronium, where the $\sim 10$ ps lifetime could be measured for the first time; iv) Thomson scattering on the accelerated electrons could provide a new source of incoherent or coherent $\mathrm{x}$-ray pulses; v) Last but not least, the possibility to perform relativistic nonlinear optics on a millijoule system makes this nascent field accessible to a much wider scientific endeavor.

\section{LASER-PLASMA MATCHING}

It is convenient to express the laser field amplitude in terms of normalized vector potential $a=\frac{e A}{m c^{2}}$, where $A$ is the laser field vector potential, $e$ the charge of the electron, $m$ the electron mass and $c$ the speed of light. The value of $a$ can be obtained from the expression $I \lambda^{2}=1.37 \cdot 10^{18} \mathrm{~W} / \mathrm{cm}^{2} \cdot a^{2}$, where $I$ and $\lambda$ are the laser intensity and wavelength. It is generally considered that the relativistic intensity threshold is reached for $a=1$, corresponding for $\lambda=800 \mathrm{~nm}$ to $2.10^{18} \mathrm{~W} / \mathrm{cm}^{2}$. We will also define the relativistic factor as $\gamma=\left(1+a^{2} / 2\right)$. The intensity or $\gamma$ distribution across the beam will lead to a mass change and a plasma frequency radial distribution. These effects will produce a radial distribution in plasma index of refraction with a maximum on axis leading to self-focusing. The self-focusing will shrink the size of the laser beam to a single wavelength transverse dimension, increasing the laser intensity accordingly. The threshold for the self-focusing power is given by the expression?:

$$
P_{c}=16.2\left(\omega / \omega_{p 0}\right)^{2} \text { in } \mathrm{GW},
$$

where $\omega_{p 0}=\left(4 \pi n_{o} e^{2} / m\right)^{1 / 2}$ is the plasma frequency at low intensity. 
In order to avoid beam break up, we propose to match the numerical aperture of the input optics to the numerical aperture of the relativistic channel. Using the plasma index of refraction given by

$$
n(r)=\sqrt{1-\omega^{2}(r) / \omega^{2}},
$$

where $\omega_{p}=\left(4 \pi n_{o} e^{2} / m\right)^{1 / 2}$ is the plasma frequency with relativistic correction.

The output of our laser ${ }^{8}$ is approaching $1 \mathrm{~mJ}$ in $10 \mathrm{fs}$ or $100 \mathrm{GW}$. From equation (1) we find that a value of $\left(\omega / \omega_{p o}\right)^{2} \approx 5$, is necessary to reach relativistic self-focusing. A Taylor expansion of (2) gives

$$
n(r) \approx 1-\omega^{2}{ }_{p}(r) / 2 \omega^{2}
$$

Note that (3) is the expression of a simple quadratic graded index waveguide if $\omega_{p}$ is proportional to $r$. This condition is fulfilled, for a Gaussian beam described by $I(r)=I_{0} \exp \left(2 r^{2} / \sigma_{o}^{2}\right)$, where $\sigma_{0}$ is the laser beam size. A graded index channel waveguide, with the index profile

$$
n(r)=1-\beta^{2} r^{2} / 2
$$

has a numerical aperture $N A=\beta b$ where $b$ is the fiber aperture. By a proper identification between (3) and (4), we can derive the channel numerical aperture given by $N A \approx \omega_{p 0} / \omega$. For $\left(\omega / \omega_{p 0}\right)^{2} \approx 5$, we find a $N A \sim 0.4$, corresponding to a waveguide diameter of about $\lambda$ or $0.8 \mu \mathrm{m}$ and $n_{0}=2.10^{20} \mathrm{~cm}^{-3}$. As we have shown this spot size could be obtained by using a deformable mirror and f/1 paraboloid. The channel $N A$ will determine, for a given laser wavelength, the plasma density for the rest of the experiments.

To demonstrate the importance of optimum laser-plasma matching we performed simulations with the use of the 2D-3V PIC code ${ }^{10}$. The laser pulse with the wavelength $\lambda$ $=0.8 \mu \mathrm{m}$, the intensity $\mathrm{I}=5.10^{19} \mathrm{~W} / \mathrm{cm}^{2}(\mathrm{a}=4.8)$, and the pulse-duration $\tau=20 \mathrm{fs}$, which is linearly polarized in the $(x, y)$-plane (p-polarized light) incidents on an underdense plasma slab placed at $10 \lambda<x<50 \lambda$. The initial plasma density is $n_{e}=0.5 n_{c}$ and the ion to electron mass ratio is 1836 . Figure 1 shows laser pulse propagation in a plasma when it is focused at the plasma-vacuum interface, $x=10 \lambda$ with a $1 \lambda$ density gradient. This focusing corresponds to the optimum conditions for the laser-plasma matching. Just behind the focus the laser pulse becomes guided due to the relativistic self-focusing, as it is seen in Fig. 1 which shows the distribution of the electromagnetic energy density in the $(x, y)$ plane. At $t=50(2 \pi / \omega)$ the laser pulse loses almost all its energy. The ponderomotive pressure of the light forms the channel in plasma. The laser pulse accelerates electrons predominantly in the forward direction and ions in the transverse direction. The maximum electron energy is about $12 \mathrm{MeV}$. The maximum ion energy reaches $\varepsilon_{i, \max } \sim 0.5$ $\mathrm{MeV}$. This is close to the estimation that follows from the mechanism of "Coulomb explosion"11 for the channel with diameter $d \sim \lambda, \varepsilon_{i, \max } \approx\left(m_{e} / m_{i}\right)(\gamma c \tau / d)^{2} \mathrm{MeV} \approx 0.4$ 
$\mathrm{MeV}$. We observed, as well, that nonlinear ion wave breaking also contributes to the ion acceleration.

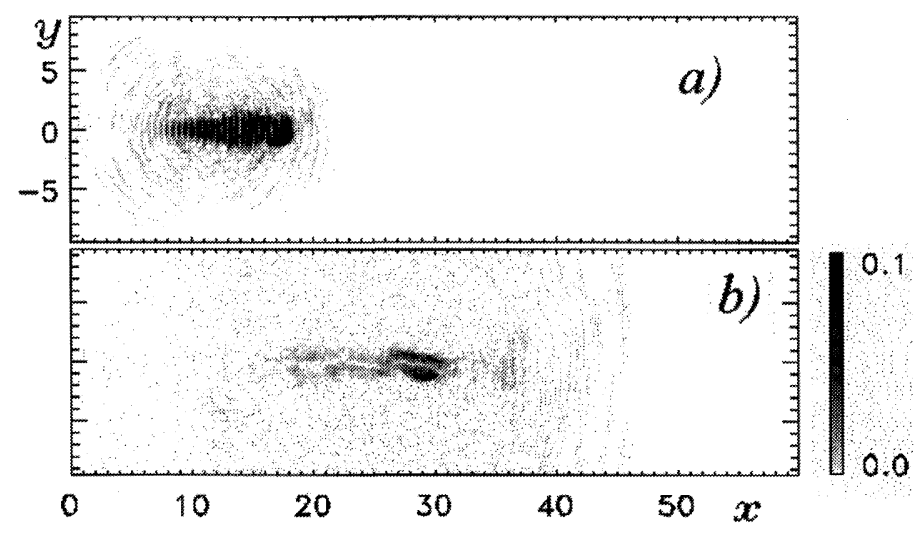

Figure 1. Electromagnetic energy density in the (x,y)-plane at $t=30(2 \pi / \omega)$ (a) and $60(2 \pi / \omega)(b)$ for optimum laser-plasma matching

Figure 2 demonstrates the case of non-optimum laser-plasma matching when laser pulse is focused at the distance $5 \lambda$ inside the plasma. Just behind the focus the break up of the laser pulse into several filaments appears. As a result the laser pulse energy depletion is much stronger than in the previous case. In addition, instead of well-formed channel several relatively short and wide channels appear.

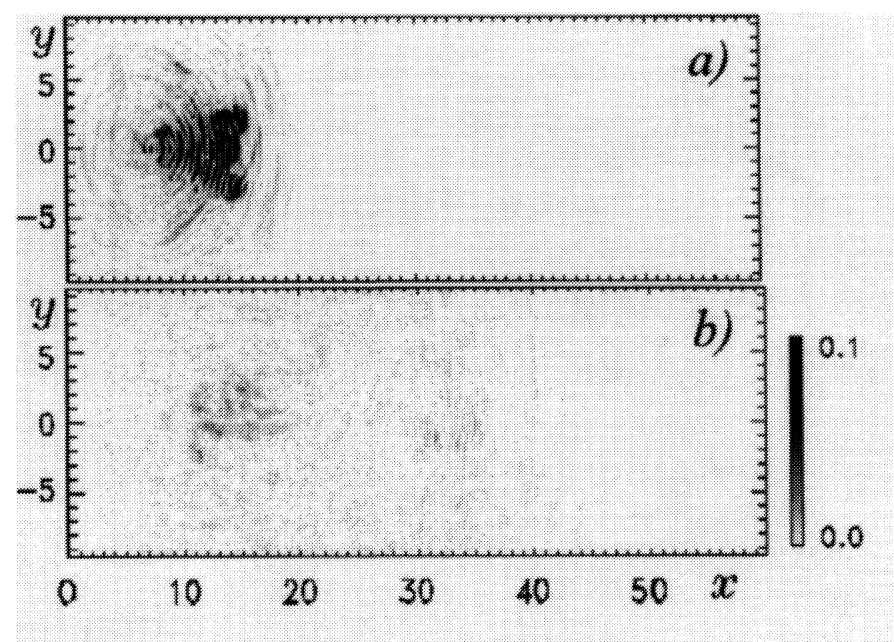

Figure 2. Electromagnetic energy density in the (x,y)-plane at $t=30(2 \pi / \omega)$ (a) and $60(2 \pi / \omega)(b)$ for non-optimum laser-plasma matching 
These features observed in PIC simulations correlate well with observations of laser channel produced in gas jet. This experiment was performed with a $400 \mathrm{fs}$ laser pulses at $\lambda=1 \mu \mathrm{m}$. Laser beam focused to a spot size of about $10 \mu \mathrm{m}$ with a paraboloidal mirror $\mathrm{f} / 3.3$ interacted with He gas jet with density $\mathrm{n}_{\mathrm{e}} \sim 0.08 \mathrm{n}_{\mathrm{c}}$. The experimental setup was described in Ref. [11]. Figure 3 shows shadowgrams of a He plasma in the defocusing (a) and relativistic self-focusing and self-channeling dominated regimes (b) for the laser intensity of $6.10^{18} \mathrm{~W} / \mathrm{cm}^{2}$ and different distances from the nozzle top. Increase in the distance from the nozzle top corresponds to a less sharp vacuum-gas interface, which leads to a breaking of laser-plasma matching conditions. The defocusing dominated regime is characterized by the formation of a short on-axis channel and offaxial laser filamentation. On other hand the regime of relativistic self-channeling characterized by the trapping of significant portion of the laser beam into a long axial plasma channel, which extends to the end of the gas jet.
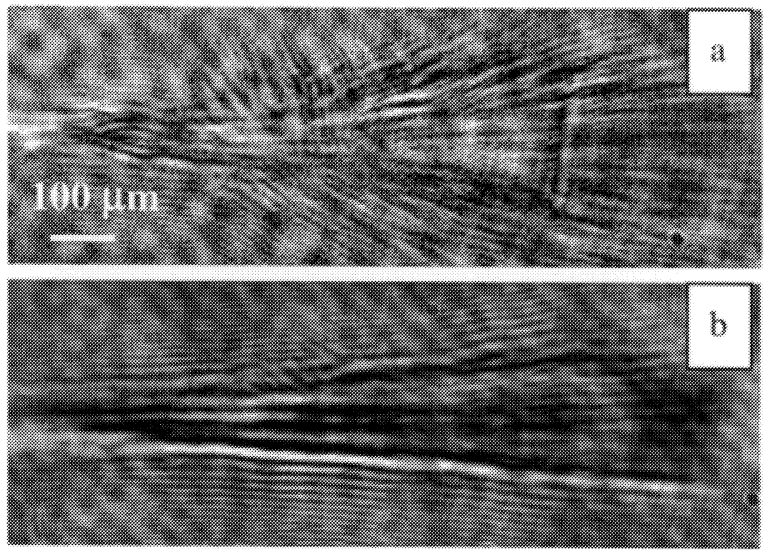

Figure 3. Shadowgrams of laser interaction with a He jet target for laser beam focused at a distance $1.5 \mathrm{~mm}$ (a) and 0.5 (b) from the top of supersonic gas nozzle. The probe beam is orthogonal to the pump beam and delayed by $10 \mathrm{ps}$. The high-intensity laser beam propagates from the left to the right. Relativistic channel is clearly visible on (b) as a bright on-axis line. An external plasma cone is formed due to He gas ionization by the spatial wings of the laser beam.

Our second example deals with laser-plasma matching at the front of solid target. Particularly, we discuss here ion acceleration in an overdense plasma in the interaction of the p-polarized laser pulse $\left(\lambda=0.8 \mu \mathrm{m}, \mathrm{I}=10^{20} \mathrm{~W} / \mathrm{cm}^{2}(\mathrm{a}=4.8), \tau=20 \mathrm{fs}\right)$ with the aluminum foil. The foil of the thickness $0.8 \mu \mathrm{m}$ and the density $\mathrm{n}=6.5 \mathrm{n}_{\mathrm{c}}$ precedes with the low density plasma layer of $5.2 \mu \mathrm{m}$ length, where the density rises up exponentially from zero 
to the critical density at the front side of the foil, $x=10 \lambda$. Laser light is focused on the front side of the foil into the spot with diameter $0.8 \mu \mathrm{m}$. In Fig. 4 we show the distribution of the electron (a) and ion (b) densities in the $(x, y)$-plane, and the ion phase plane $\left(p_{x}, x\right)$ in frame (c) at $\mathrm{t}=200(2 \pi / \omega)$. We can see that the maximum ion energy gain is about $48 \mathrm{MeV}$.

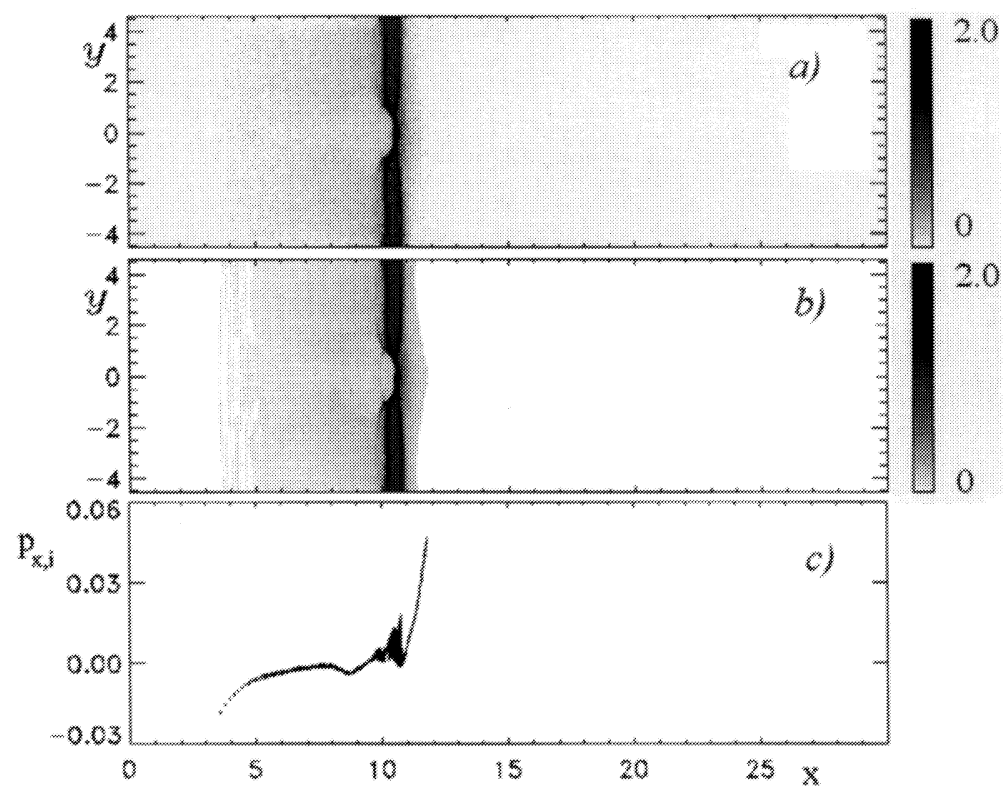

Figure 4. Results of the PIC simulations of the interaction of the $20 \mathrm{fs}$ p-polarized laser pulse $(a=6.8)$ with a thin slab of overderdense $\left(n / n_{c}=6.5\right)$ plasma at $t=200(2 \pi / \omega)$. The distribution in the $(x, y)$-plane of the electron (a) ion (b) density, and the ion phase plane $\left(p_{x}, x\right)(\mathrm{c})$.

For the past ten years research on short-laser-pulse interaction with plasmas has basically striven for higher laser energy. Very little efforts have been devoted to the control of the laser-plasma matching. Here we show that this control allows to achieve the highest parameters for channeling and high-energy particle generation. 


\section{ACKNOWLEDGMENTS}

This research was supported by the National Science Foundation through the Center for Ultrafast Optical Science, contract STC PHY8920108 and the Russian Foundation for Basic Research, grant No. 00-02-16063.

\section{REFERENCES}

1. G. A. Mourou, C. P. J. Barty, and M. D. Perry, Physics Today, January 1998, 22-28.

2. D. Umstadter, S. -Y. Chen, A. Maksimchuk, G. Mourou, and R. Wagner, Science 273, 472-475 (1996).

3. A. Maksimchuk, S. Gu, K. Flippo, D. Umstadter, and V. Yu. Bychenkov, Phys. Rev. Lett. 84, 4108-4111, (2000).

4. P. Norreys, M. Santala, E. Clark, M. Zepf, I. Watts, F. N. Beg, K. Krushelnick, M. Tatarakis, A. E. Dangor, X. Fang, P. Graham, T. McCanny, R. P. Singhal, K. W. D. Ledingham, A. Creswell, D. C. W. Sanderson, J. Magill, A. Machacek, J. S. Wark, R. Allott, B. Kennedy, and D. Neely, Phys. Plasmas 6, 2150-2156 (1999).

5. D. von der Linde, T. Engers, and G. Jenke, P. Agostini, G. Grillon, E. Nibbering, A. Mysyrowicz, and A. Antonetti, Phys. Rev. A 52, R25-7 (1995).

6. A. B. Borisov, A. V. Borovskiy, O. B. Shiryaev, V. V. Korobkin, A. M. Prokhorov, J. C. Solem, T. S. Luk, K. Boyer, and C. K. Rhodes, Phys. Rev. A 45, 5830-5845 (1992).

7. S. -Y. Chen, A. Maksimchuk, and D. Umstadter, Nature 396, 653-655 (1998).

8. O. Albert, H. Wang, D. Liu, Z. Chang, and G. Mourou, Opt. Lett. 25, 1125-1127 (2000).

9. G.-Z. Sun, E. Ott, Y. C. Lee, and P. Guzdar, Phys. Fluids 30, 526-532 (1987).

10. T. Zh. Esirkepov, Comput. Phys. Comm. 135, 144-53 (2001).

11.G. S. Sarkisov, V. Yu. Bychenkov, V. N. Novikov, V. T. Tikhonchuk, A. Makismchuk, S. -Y. Chen, R. Wagner, G. Mourou, and D. Umstadter, Phys. Rev. E 59, $7042-7054$ (1999). 